\title{
Circunferencia de la cintura como predictor de insulinoresistencia
}

\section{Objetivo}

Determinar la efectividad de las mediciones antropométricas y de los marcadores bioquímicos para predecir la sensibilidad a la insulina.

\section{Diseño}

Retrospectivo, observacional de corte transversal.

\section{Lugar \\ Departamento de Medicina y Departamento de Fisiología Clínica de Karolinska Institutet, Karolinska University Hospital, Suecia.}

\section{Pacientes}

Muestra de 2746 voluntarios sanos (798 hombres) de 18 a 72 años, índice de Masa Corporal (IMC) 18 a $60 \mathrm{~kg} / \mathrm{m}^{2}$ y circunferencia de cintura de 65 a $150 \mathrm{~cm}$.

\section{Descripción de la prueba en estudio y de la de referencia}

Se determinó el peso, la talla, la circunferencia de la cintura (medida en el punto medio entre la última costilla y la cresta ilíaca) y la circunferencia de la cadera. Se midió glucosa, insulina, lípidos y leptina. Se calculó el índice HOMA como medida de sensibilidad a la insulina [glucosa $(\mathrm{mmol} / \mathrm{L}) \mathrm{x}$ insulina $(\mathrm{mU} / \mathrm{L}$ ] $/ 22,5)$. Se definió insulinorresistencia al puntaje HOMA mayor a 3,99.

Se utilizó un modelo multivariable* de regresión para establecer el valor predictivo de las variables y se realizó análisis de la curva $\mathrm{ROC}^{*}$ para establecer un punto de corte apropiado de las variables estudiadas.

\section{Resultados principales}

EI IMC fue $32,4 \pm 9,4 \mathrm{~kg} / \mathrm{m}^{2}$ en hombres y $31,6 \pm 9,3 \mathrm{~kg} / \mathrm{m}^{2}$ en mujeres; la circunferencia de la cintura fue $109,3 \pm 25,5$ y $97,9+21,6 \mathrm{~cm}$ en hombres y mujeres respectivamente. La circunferencia de cintura fue el factor que tuvo mayor valor predictivo Las características operativas de una circunferencia mayor o igual a $100 \mathrm{~cm}$ aparecen en el cuadro 1.

Cuadro 1. Características operativas de una circunferencia mayor o igual a $100 \mathrm{~cm}$

\begin{tabular}{|c|c|c|c|c|c|c|c|}
\hline \multirow[b]{2}{*}{ Grupo } & \multirow{2}{*}{$\begin{array}{c}\text { Sensibil. \% } \\
\text { IG95\% }\end{array}$} & \multirow{2}{*}{$\begin{array}{c}\text { Especif. \% } \\
\text { IC95\% }\end{array}$} & \multicolumn{2}{|c|}{$\begin{array}{c}\text { Valor } \\
\text { predictivo }\end{array}$} & \multicolumn{2}{|c|}{$\begin{array}{l}\text { Coeficiente } \\
\text { de probabil. }\end{array}$} & \multirow{2}{*}{$\begin{array}{c}\text { Area } \\
\text { bajo la } \\
\text { curva } \\
\text { ROC }\end{array}$} \\
\hline & & & $\begin{array}{c}\text { Neg. } \\
\%[\text { G95\% }\end{array}$ & \begin{tabular}{|c|} 
Pos. \\
$\%$ [C95 $\%$ \\
\end{tabular} & Pos. & Neg. & \\
\hline Hombres & $98(95-99)$ & $63(59-68)$ & 98(96-99) & $61(56-66)$ & 2,6 & 0,04 & $89 \%$ \\
\hline Mujeres & $94(91-96)$ & $63(61-66)$ & $97(96-98)$ & $42(38-45)$ & 2,56 & 0,10 & $86 \%$ \\
\hline
\end{tabular}

\section{Conclusiones}

La circunferencia de la cintura es un buen predictor de sensibilidad a la insulina y un valor menor a $100 \mathrm{~cm}$ permite descartar insulinorresistencia en ambos sexos.

Fuente de financiamiento: Swedish Research Council, Swedish Diabetes Association, Novo Nordic Foundation, Swedish Heart and Lung Foundation y Karolinska Institute

\section{Comentario}

La obesidad abdominal es un factor predictor de síndrome metabólico y de enfermedad cardiovascular. El perímetro de cintura se correlaciona con insulinorresistencia, hipertensión y dislipemia, siendo mejor predictor que el IMC ${ }^{1,2}$.

Con el aumento de la obesidad intrabdominal, aumenta la liberación de ácidos grasos al hígado por la circulación esplácnica, lo que aumenta la disponibilidad de glucosa y gluconeogénesis, inhibe el clearance hepático de la insulina aumentando el hiperinsulinismo e incrementa la producción de lipoproteínas VLDL con aumento de la Apo B100, que posteriormente dará origen a las LDL pequeñas y densas. El tejido adiposo es además fuente de citoquinas inflamatorias (TNFa, IL6 y PCR), incrementadas del síndrome metabólico ${ }^{3}$.

La obesidad central es uno de los pilares diagnósticos del síndrome metabólico, ya sea una relación cintura/cadera mayor a 0,9 en hombres ó mayor a 0,85 en mujeres según criterios de la OMS, como el perímetro de cintura mayor a 102 y $88 \mathrm{~cm}$ en hombres y mujeres, respectivamente según los criterios NCP ATP III. Una circunferencia mayor a $88 \mathrm{~cm}$ en mujeres implica una especificidad de $49 \%{ }^{4}$. Recientemente, la Federación Internacional de Diabetes ${ }^{5}$ ha consensuado una nueva definición en la que la obesidad central es un criterio excluyente y requiere además, dos o más de los si- guientes factores: 1) trigliceridemia mayor a $150 \mathrm{mg} / \mathrm{dL}$; 2) colesterol HDL menor a $40 \mathrm{mg} / \mathrm{dL}$ en hombres y $50 \mathrm{mg} / \mathrm{dL}$ en mujeres; 3 ) tensión arterial mayor a $130 / 85 \mathrm{mmHg}$; 4) glucemia en ayunas mayor a $100 \mathrm{mg} / \mathrm{dL}$ o diagnóstico de diabetes. En este consenso la obesidad central es el criterio mayor y esta definido por un perímetro de cintura mayor a $94 \mathrm{~cm}$ en hombres y a $80 \mathrm{~cm}$ en mujeres para los europeos, con distintos niveles de cortes según las etnias.

En este trabajo los autores observan que una circunferencia de cintura menor a $100 \mathrm{~cm}$ excluye en ambos sexos el riesgo de insulinoresistencia, definida como HOMA mayor de 3,99. Sin embargo, este nivel de corte de cintura a $100 \mathrm{~cm}$, implica una menor sensibilidad para detectar una serie de pacientes con riesgo cardiovascular aumentado, principalmente mujeres.

\section{Conclusiones del comentador}

La medición del perímetro de cintura es un parámetro clínico útil y de fácil realización en la práctica clínica cotidiana; permite detectar aquellos pacientes con riesgo de insulinorresistencia, síndrome metabólico y alto riesgo cardiovascular.

\section{Juliana Fassi [ Servicio de Endocrinología y Medicina Nuclear. Hospital Italiano de Buenos Aires. ]}

Farsi J. Wahrenberg H, Hertel K, Leijonhufvud BM y col. La circunferencia de la cintura es un buen predictor de insulinoresistencia. Evid actual pract ambul 2005;8:139. Disponible en URL: www.evidencia.org Use of waist circunference to predict insulin resistance: retrospective study. BMJ 2005;330:1363-4. PMID: 15833749

\section{Referencias}

1. Ford E S, Giles W H, Dietz W H. Prevalence of the Metabolic Syndrome among US adults. JAMA.2002.287:356-359

2. Lofgren I, Herron K, Zern T et al. Waist circunference is a better predictor than Body Mass Index of Coronary Heart Risk in Overweight Premenopausal Women. J nutr.2004.134:10711076 .

3. Eckel R, Grundy S M, Zimmet P Z. The Metabolic syndrome. The Lancet.2005.365:1415-1428.

4. Han T S, Leer E M, Seidell J C, Lean M E, Waist circunference action levels in the identification of cardiovascular risk factors: prevalence study in a random sample. BMJ.1995 311:1401-

4. Han T S, Leer E M, Seidell J C, Lean M E, Waist circunference action levels in the identification of cardiovascular risk fact
1405 .

5. The IDF (International Diabetes Federation) concensus worldwide definition of the metabolic syndrome. www.IDF.org 